\title{
Application of computational methods for harmonic state estimation of power system networks
}

\author{
Hassan Saadallah Naji ${ }^{1}$, Husham Idan Hussein ${ }^{2}$ \\ ${ }^{1}$ Department of Biomedical Engineering, College of Engineering, kerbala University, Iraq \\ ${ }^{2}$ Department of Electrical power and Machines Engineering, College of Engineering, University of Diyala, Iraq
}

\begin{tabular}{|c|c|}
\hline Article Info & ABSTRACT \\
\hline Article history: & In this study, a novel technique is used to estimate the power system \\
\hline Received Aug 26, 2020 & harmonic state, as one of the biggest risks in a power system network. \\
\hline Revised Nov 29, 2020 & injected harmonics make networks unstable and increase power loss. The \\
\hline Accepted Dec 9, 2020 & $\begin{array}{l}\text { main objective of this work is to develop a new harmonic state estimator } \\
\text { system to increase power system accuracy, stability and the wall operation }\end{array}$ \\
\hline Keywords: & $\begin{array}{l}\text { state. Three computational methods are used in this study, that is, the i) } \\
\text { proposed particle swarm optimisation-recursive least squares (PSO-RLS) }\end{array}$ \\
\hline Computational methods & algorithm, which is developed, presented and compared with the ii) discrete \\
\hline Harmonics & $\begin{array}{l}\text { fourier transform (DFT) and iii) PSO algorithms. The three algorithms are } \\
\text { tested on an IEEE 14-bus system, and simulation results show that the new }\end{array}$ \\
\hline Hybrid algorithm & PSO-RLS algorithm is more accurate than the other two algorithms (i.e. DFT \\
\hline Power system & and PSO algorithms), with a lower error percentage. The proposed algorithm \\
\hline PSO & is tested to prove its validity and effectiveness in power system networks. \\
\hline RLS & The capability of the PSO-RLS algorithm is apparent in the error percentage \\
\hline State estimation & $\begin{array}{l}\text { compared with that of the other two computational methods, which can be } \\
\text { used to provide an excellent prediction rate for measurement errors in system } \\
\text { buses. }\end{array}$ \\
\hline
\end{tabular}

This is an open access article under the CC BY-SA license.

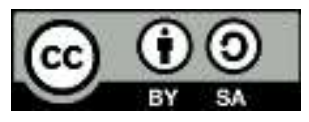

Corresponding Author:

Hassan Saadallah Naji

Department of Biomedical Engineering

College of Engineering

kerbala University, Iraq

Email: Hassan.saadallah@uokerbala.edu.iq

\section{INTRODUCTION}

The power system harmonics' study is of considerable interest to power system researchers owing to the importance and direct effect of power provided to consumers. Harmonics in power systems are proportional to power system quality and reliability, that is, if harmonics increase, then quality and reliability decrease. For example, nonlinear loads (e.g. power electronics circuits, adjustable speed drives, power supplies, silicon controlled rectifiers and so on) inject harmonics or can be considered as sources of harmonics in power system networks. Numerous researchers are interested in harmonics and thus find and develop algorithms that can help calculate and predict harmonics in a power system network.

A hybrid algorithm is used to estimate harmonics and amplitudes in a Kalman filter (KF) by applying a linear estimator based on least squares and solving a nonlinear equation (iterated extended KF) that describes the waveform with a phase. The least-squares and recursive least-squares (RLS) approaches used to predict amplitudes are shown in [1]. Discrete Fourier transform (DFT) is one of the most important techniques employed for waveform analysis [2-5]. However, major limitations of the periodic technical 
method are explicit aliasing, leakage and picket-fence errors [6], which are the main challenges encountered by researchers in this field. Validation of a hybrid algorithm for state estimation is achieved with satisfactory performance rates [7]. Real power system load models are nonlinear and linear in phase and amplitude, respectively [8]. Nonlinear loads encounter problems with algorithm convergence speed; therefore, hybrid algorithms are used to estimate phase and amplitude values inside each load to avoid such problems [9]. In [10], FACTS devices were utilised to improve power system operation control, and PMU was used as a monitor to improve the capability of state estimation systems and full observable systems. The stability of a power system is a crucial area of research to describe the harmonics problem, and many studies employ FACT devices, such as DSTATCOM, as shown in [11, 12]. State estimation is extremely important in a power distribution system for determining the variable state in a system network, as shown in [13].

The main purpose of monitoring a system is to observe and protect a full system. However, such a system cannot monitor harmonics with a traditional meter; thus, a state estimation method is crucial for power systems. Moreover, evolutionary algorithms are also introduced, which can describe monitoring system state variables in a power network (current and voltage) [14]. Topology evaluation networks were investigated in [15], which are defined as strategic sites for meter connections, and measured values used to calculate the rest of the variable state (X series and $\mathrm{Y}$ shunt). In [16, 17], the topology approach was introduced and used to achieve harmonic state estimation (HSE), which is conducted by assuming that the current measured by a power quality meter is connected to a bus and can change the PQ meter location depending on the number of sections linked to the corresponding bus. However, the assumptions in this approach are not pragmatic owing to the limited number of channels for most power quality meters. Thus, amendments are made to permit the use of the topology approach to obtain optimum locations for installing power quality meters. Contingent on system availability, when a set number of channels are considered for power quality meters, a few association options become accessible at an established site. In this way, the system would characterise how the meters should be associated with a power arrangement rather than their number and establishment destination [18].

Finally, two methods are used in this study (i.e. discrete fourier transform DFT and particle swarm optimisation PSO) and compared with the proposed novel PSO-RLS method. MATLAB is employed to achieve the objective of the study, and the computational method is tested on an IEEE 14-bus standard system. The results show that the PSO-RLS method is satisfactory, fast and more accurate than the other two methods. This procedure can upgrade the number of estimations and limit estimation mistakes simultaneously. Based on the above information, the main contributions of this study are as follows:

a) Application of computational methods, such as the hybrid algorithm (PSO-RLS), for state estimation; all fundamental, inter- and subharmonics of power system signals contain various noise sources.

b) Evaluation of two important parameters of the proposed PSO-RLS algorithm, that is, the performance and best harmonic state estimator; the proposed algorithm is then compared with common DFT and PSO algorithms.

c) Evaluation of the three algorithms' performance in estimating the harmonic parameters of standard system data precisely for finding the best and most appropriate method for harmonic estimation.

The rest of this paper is organised as follows. In Section 2, the definitions of general power system harmonics are given, and in Section 3, the state estimation formulation is provided. In Section 4, the mathematical background of the study is explained, and in Section 5, the computational PSO-RLS method is described. The results and discussion are presented in Section 6, and the conclusion is provided in Section 7.

\section{POWER SYSTEM HARMONICS}

In power system networks, the harmonics problem is a research hotspot for power system scholars; Therefore, harmonics analysis, specifically, fourier transform, plays a substantial role in engineering studies on fourier series development. Episodic and nonsinusoidal source currents contain or consist of a series of sinusoidal harmonics; thus, the response to each harmonic can be determined by the following [19]:

$$
f(t)=A_{0}+\sum_{n=1}^{\infty}\left(A_{n} \sin n t+B_{n} \cos n t\right)
$$

Where $n=1,2,3$ is the harmonic order, $t$ is the time variable and $A 0, \ldots, A n$ and $B 1, \ldots, B n$ are the harmonic constants. The total harmonic distortion factor is considered as the most common identification indicator for the existence of harmonics in a signal, which for voltage and current signal sources can be obtained (2) and (3): 


$$
\begin{gathered}
T H D_{v}=\frac{\sqrt{\sum_{n=2}^{\infty} v_{n}^{2}}}{v 1} \\
T H D_{I}=\frac{\sqrt{\sum_{n=2}^{\infty} I_{n}^{2}}}{I 1}
\end{gathered}
$$

where $V I$ and $I I$ are the fundamental component's root mean square (RMS) value for voltage and current signal, respectively, and $V n$ and $I n$ are the $n t h$ component's RMS value for voltage and current signal, respectively $[20,21]$.

$$
\begin{aligned}
& V(t)=x_{0} e^{-t \lambda}+\sum_{i=1}^{N} A_{c, i} \cos (i w o t+\theta c, i)+A_{s, i} \operatorname{Sin}(i w o t+\theta s, i) \\
& I(t)=x_{0} e^{-t \lambda}+\sum_{i=1}^{N} A_{c, i} \cos (i w o t+\theta c, i)+A_{s, i} \operatorname{Sin}(i w o t+\theta s, i)
\end{aligned}
$$

where $x_{0}$ is the signal's constant component, $\lambda$ is signal's time constant and $A c, i, A s, i, \theta c, i$ e $\theta s, i$ are amplitudes and phase angles of the ith harmonic of sinusoidal terms. In addition, $w_{0}$ is the frequency fundamental component, $i$ is the order of the harmonic components and $\mathrm{N}$ is the total number of harmonics, which is used to express $x(t)$.

\section{STATE ESTIMATION FORMULATION}

The following mathematical equation shows the mathematical relationship between the measurements and estimated value and error $[13,22]$.

$$
Z_{i}=h(x i)+e_{i}, i=1,2,3,4, \ldots m
$$

where $e_{i}$ is the ith error in the measurement, $h(x i)$ is the function that relates the state variables with the measurements, $m$ is the measurements' numbers and $x$ is the state variable (all bus angles and voltages, excluding the slack bus angle in this case).

\section{MATHEMATICAL BACKGROUND}

\subsection{DFT}

Fourier analysis consists of various types of signal processing, such as fourier transformation and DFT, which is a type of discrete transformation that involves a finite sequence of discrete data equivalent to the continuous fourier transform of signals known only at instants separated by sample times.

Let $f(t)$ be a continuous signal (data source).

Let $N$ be $f[0], f[1], f[2] \ldots . ., f[k], \ldots f[N-1]$

$F(j w)$ is a Fourier Transform of the original signal, which can be written as:

$$
F(j w)=\int_{-\infty}^{\infty} f(t) e^{-j w t} d t
$$

We can respect each example $f[k]$ as a drive with zone $f[k]$. At this point, given that the integrand exists only at the sample focuses,

$$
F(j w)=\int_{0}^{(N-1) \tau} f(t) e^{-j w t} d t=f[0] e^{-j 0} d t+f[1] e^{-j w t} d t+\cdots+f[k] e^{-j w k T}+\cdots f(N-1) e^{-j(N-1) \tau}
$$

that is,

$$
F(j w)=\int_{k=0}^{N-1} f[k] e^{-j w k T}
$$

On a fundamental level, we can assess this for any w, yet with only information focuses, initially, only last yields are crucial [23-25].

\subsection{Particle swarm optimization state estimation}

PSO is an improvement procedure that models social manner and utilises a populace of particles to look inside an enquiry area using a multidimensional mode. In the hunt space inside a specified time interim, 
every molecule possesses its own position, and the speed of the movement of such molecules is altered according to the sum of experiences picked up by the molecules with the experiences of the different particles in the gathering (swarm). Each molecule's experience incorporates pertinent data from its direction in the hunt space for better storage in its best previously involved position [26]. Therefore, each molecule's best previously involved position relates to learning retention associated with great arrangements acquired during the iterative procedure.

$$
\begin{aligned}
& v_{i d}(t+1)=v_{i d}(t)-c_{1} * \operatorname{rand}() * \\
& {\left[p_{i d}(t)-x_{i d}(t)\right]+c_{2} * \operatorname{rand}() *\left[p_{g d}(t)-x_{i d}(t)\right]} \\
& x_{i d}(t+1)=x_{i d}(t)+v_{i d}(t+1), 1 \leq i \leq n, 1 \leq d \leq D
\end{aligned}
$$

Where $\operatorname{rand}()$ is a random number between 0 and $1 ; c 1$ and $c 2$ are the positive values of acceleration constants, where $c 1$ and $c 2$ are 1.5 and 2.5, respectively; and $w$ is the inertia weight. Implementation of the algorithm requires placing a limit on the velocity ( $V$ max $)$ in addition to the acceleration constants. The PSO can attain its best search capabilities after the w and (Vmax) parameters are adjusted.

$$
\begin{aligned}
& v_{i d}(t+1)=w^{*} v_{i d}(t)+c_{1} * \operatorname{rand}() * \\
& {\left[p_{i d}(t)-x_{i d}(t)\right]+c_{2} * \operatorname{rand}() *\left[p_{g d}(t)-x_{i d}(t)\right]} \\
& x_{i d}(t+1)=x_{i d}(t)+v_{i d}(t+1) ; 1 \leq i \leq n_{\&} 1 \leq d \leq D
\end{aligned}
$$

The basic of the PSO technique is defined as shown in Figure 1.

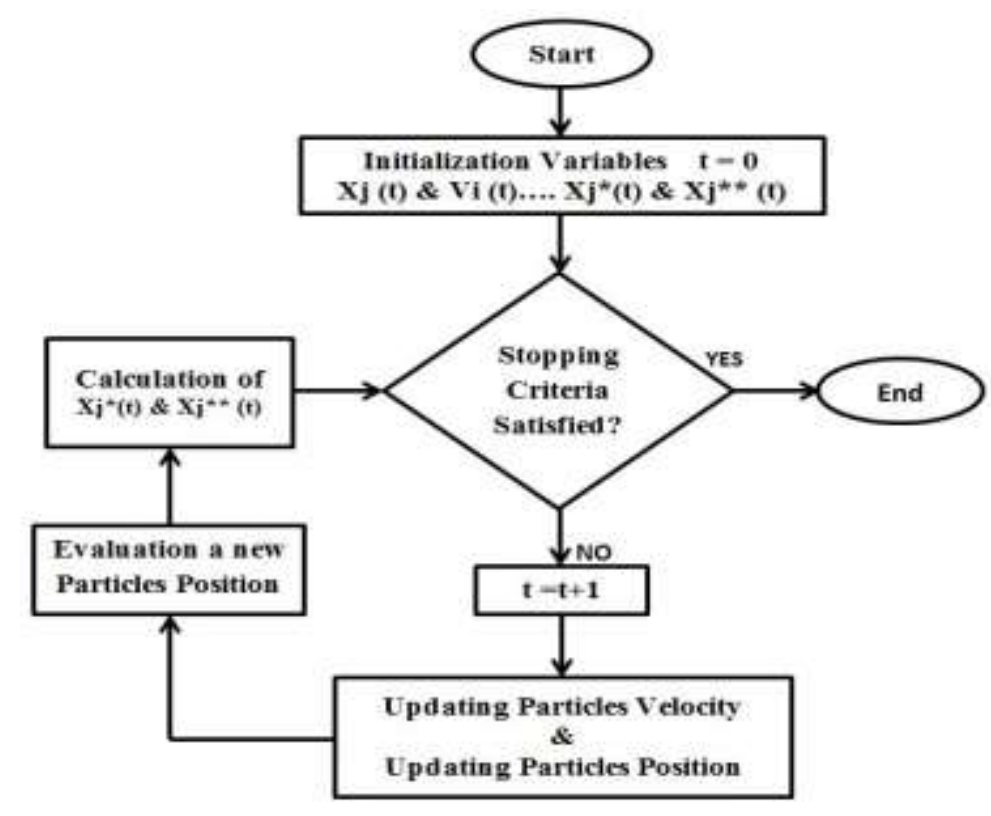

Figure 1. PSO algorithm flow chart

\section{COMPUTATIONAL PARTICLE SWARM OPTIMIZATION-RECURSIVE LEAST SQUARE (PSO-RLS)}

The procedure for the computational PSO-RLS algorithm is as follows:

1. The standard PSO algorithm is used to achieve optimisation for the unknown parameters.

2. The output of step 1 is used to create the initial values of the RLS algorithm.

3. A comparison is conducted between the output of step 2 and the desired output.

4. The weights of the RLS responsible for minimising error in the actual output are adjusted (updated). 


\section{RESULTS AND DISCUSSION}

In this study, three methods are used to estimate the harmonics of a power system, that is, the DFT (traditional), PSO and PSO-RLS (computational) methods, which are compared with the manual calculation of (4) and (5) based on the results. The three algorithms are tested and examined on an IEEE 14-bus standard system. As a balanced system, all buses except bus No. 7 (without sources) inject harmonics as a nonlinear loads (13 suspicious nodes). For the database, the authors take the measurements of current harmonics and voltage harmonics of branches and nodes, respectively. The proposed algorithm (PSO-RLS) for HSE is written with MATLAB as an estimator and optimiser for high-level harmonic components (amplitude and phase values of fundamentals) in a time fluctuating waveform. The proposed algorithm is validated according to the results and provides fast calculation and high estimation accuracy [27]. Table 1 presents the voltage harmonic percentage of each bus and results obtained by the manual calculation of (4) and (5), which are based on other results. Table 1 shows that the 5 th harmonic value of bus 1 is the biggest value.

Table 1. Voltage harmonics for each bus IEEE14bus (4)

\begin{tabular}{|c|c|c|c|c|c|}
\hline \multirow{2}{*}{ fundamental } & \multicolumn{5}{|c|}{ Hand calculation \% } \\
\hline & 5 th & 7 th & 11 th & 13th & 17 th \\
\hline 1.07 & 0.005792 & 0.0045124 & 0.0034024 & 0.0031075 & 0.0021449 \\
\hline 1.04 & 0.0071777 & 0.0054405 & 0.0032025 & 0.0022304 & 0.00049126 \\
\hline 0.9703 & 0.013202 & 0.0081566 & 0.0065388 & 0.008053 & 0.0040828 \\
\hline 0.9854 & 0.0084957 & 0.0047932 & 0.0009719 & 0.0022266 & 0.0023329 \\
\hline 1.0173 & 0.0087342 & 0.0060512 & 0.0032662 & 0.0023007 & 0.0005924 \\
\hline 1.02 & 0.0037118 & 0.0022276 & 0.0009315 & 0.0005537 & 0.0000422 \\
\hline 0.9758 & 0.0047166 & 0.0023842 & 0.0002223 & 0.00066478 & 0.00076966 \\
\hline 0.969 & 0.0040053 & 0.0020618 & 0.0004143 & 0.00011529 & 0.00026339 \\
\hline 0.981 & 0.0038896 & 0.00220399 & 0.00045356 & 0.0001023 & 0.00023464 \\
\hline 0.9428 & 0.0027327 & 0.0012883 & 0.00036595 & 0.00017728 & 0.0000162 \\
\hline 1.0641 & 0.0028041 & 0.0013013 & 0.0003593 & 0.00017044 & 0.0000197 \\
\hline 0.9457 & 0.0035623 & 0.0016315 & 0.00011052 & 0.00030353 & 0.00036458 \\
\hline 1.0541 & 0.0027041 & 0.0013113 & 0.0003693 & 0.00016044 & 0.0000187 \\
\hline 0.9459 & 0.0035633 & 0.0016325 & 0.00011062 & 0.00030343 & 0.00036468 \\
\hline
\end{tabular}

Figures 2(a), 2(b) and 2(c) illustrate the comparison between the three computational methods, that is, the DFT, PSO and proposed PSO-RLS methods, to estimate the voltage harmonic state of power system networks. Bus 1 has the largest voltage harmonic value, followed by buses 3, 4, 7, 5, 2, 14, 12, 8, 9, 6, 11, 13 and 10. In terms of percentage values, the 5th harmonic is between $(0.006042$ and 0.0029827$)$, the 7 th harmonic is between $(0.0047625$ and 0.0015383$)$, the 11 th harmonic is between $(0.0036524$ and $0.00061595)$, the 13th harmonic is between $(0.0033575$ and 0.00042728$)$ and the 17 th harmonic is between (0.00233949 and 0.0002662) for the traditional DFT method.

The results of the computational PSO method show that the order of the buses with the highest voltage harmonic percentage value is $3,4,1,7,5,2,14,12,8,9,6,11,13$ and 10 . In terms of voltage harmonic percentage values, the 5 th harmonic is between $(0.014102$ and 0.0036327$)$, the 7 th harmonic is between (0.0090566 and 0.0021883), the 11th harmonic is between $(0.0074388$ and 0.00126595$)$, the 13th harmonic is between $(0.008953$ and 0.00107728$)$ and the 17 th harmonic is between $(0.0049828$ and $0.0009162)$.

The PSO-RLS method is the third computational method used in this study, and the results demonstrate that the order of the buses with the highest voltage harmonic percentage value is $3,4,1,7,5,2$, $14,12,8,9,6,11,13$ and 10 . . For the voltage harmonic percentage values, the 5th harmonic is between (0.013442 and 0.002973), the 7th harmonic is between $(0.008397$ and 0.001528$)$, the 11 th harmonic is between $(0.006779$ and 0.000606$)$, the 13 th harmonic is between $(0.008293$ and 0.000417$)$ and the 17 th harmonic is between (0.004323 and 0.000256) for each bus. Figure 2 exhibits the error percentage of each harmonic order, and the accuracy of the proposed PSO-RLS computational method is better than that of the other two methods, with a lower voltage error percentage. Overall voltage harmonic error as shown in Figure 3.

Table 2 shows that the harmonic current percentage of each branch in the IEEE 14-bus system depends on (5). The 5th harmonic is between (0.0066786 and 0.000596), the 7th harmonic is between (0.003016 and 0.000615), the 11th harmonic is between (0.003292 and 0.003416), the 13th harmonic is between $(0.002384$ and 0.002629$)$ and the 17 th harmonic is between $(0.001263$ and 0.000234$)$.

Table 3 shows the voltage bus angle of all 14 IEEE buses with the three methods, and Table 4 presents the voltage bus angle error of each bus and method. The results indicate that the PSO-RLS method has fewer errors than the other two methods, with a percentage value of $0.17632 \%$. 


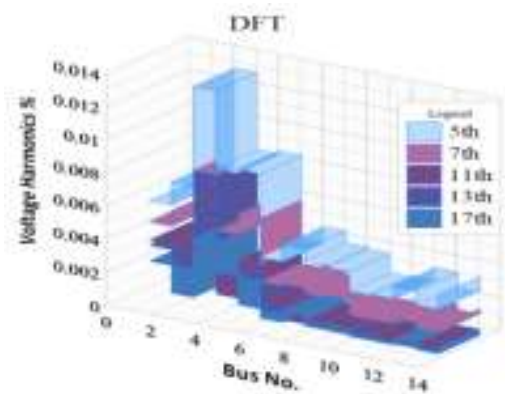

(a) DFT method

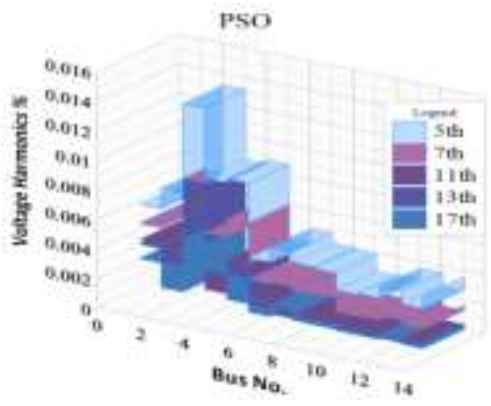

(b) PSO method

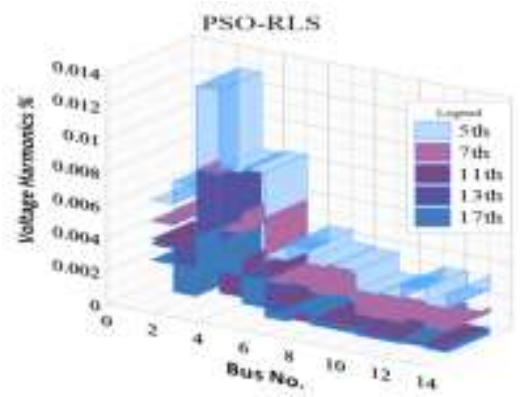

(c) PSO-RLS method

Figure 2. Voltage harmonics of each of the 14 IEEE buses; (a) DFT method; (b) PSO method; (c) PSO-RLS method

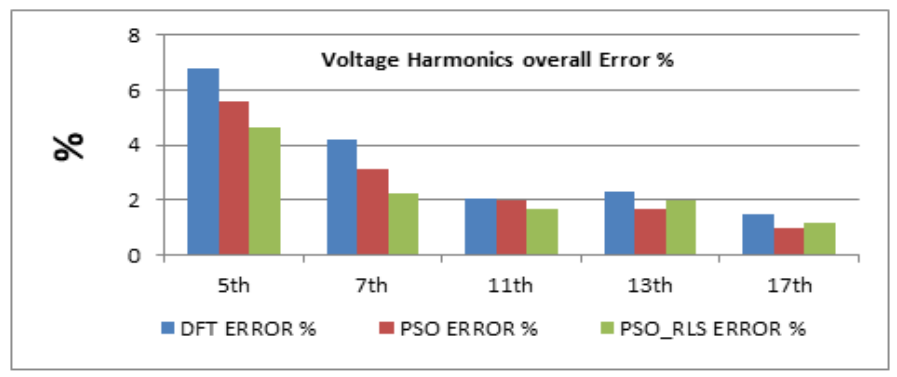

Figure 3. Overall voltage harmonic error

Table 2. Harmonic current of each branch in the IEEE 14-bus system [5]

\begin{tabular}{cccccc}
\hline branch & 5th & 7th & Hand calculation\% & 13 th & 17 th \\
\hline I1-2 & 0.0066786 & 0.003016 & 0.003292 & 0.002384 & 0.001263 \\
I1-5 & 0.008224 & 0.00323 & 0.002707 & 0.000162 & 0.000128 \\
I2-3 & 0.031552 & 0.01618 & 0.009676 & 0.01247 & 0.018209 \\
I2-4 & 0.0034164 & 0.000692 & 0.000547 & 0.002465 & 0.004287 \\
I2-5 & 0.011856 & 0.005496 & 0.002637 & 0.002666 & 0.000273 \\
I3-4 & 0.029031 & 0.018965 & 0.025964 & 0.005899 & 0.001182 \\
I4-5 & 0.0075226 & 0.005565 & 0.008078 & 0.03581 & 0.003679 \\
I4-6 & 0.000596 & 0.000615 & 0.003416 & 0.002629 & 0.000234 \\
I6-9 & 0.011354 & 0.006608 & 0.002233 & 0.002452 & 0.000742 \\
I6-10 & 0.01178 & 0.006824 & 0.002161 & 0.002411 & 0.000725 \\
I6-11 & 0.0040914 & 0.001784 & 0.003763 & 0.002447 & 0.000147 \\
I7-8 & 0.0056124 & 0.002481 & 0.00448 & 0.003116 & 0.000192 \\
I7-12 & 0.0014406 & 0.000851 & 0.004799 & 0.003801 & 0.000933 \\
I8-9 & 0.0016693 & 0.00091 & 0.004849 & 0.003851 & 0.000956 \\
I8-10 & 0.011354 & 0.006608 & 0.002233 & 0.002452 & 0.000742 \\
I9-12 & 0.000596 & 0.000615 & 0.003416 & 0.002629 & 0.000234 \\
I10-11 & 0.0014406 & 0.000851 & 0.004799 & 0.003801 & 0.000933 \\
I11-12 & 0.011856 & 0.005496 & 0.002637 & 0.002666 & 0.000273 \\
I12-13 & 0.01178 & 0.006824 & 0.002161 & 0.002411 & 0.000725 \\
I13-14 & 0.0178 & 0.0064 & 0.00211 & 0.00211 & 0.00025 \\
\hline
\end{tabular}


Figures 4(a), 4(b) and 4(c) present the current harmonic percentage of each bus in the IEEE 14-bus system for the three methods, namely, the a) DFT, b) PSO and c) PSO-RLS methods. In the PSO-RLS method, the inputs are chosen as the current, and the outputs are chosen as the magnitude of the 3rd, 5th, 7th and 11th harmonics.

Based on the comparison between the three methods and the manual calculation, the steady-state percentage error is computed and tabulated in Figure 5. The simulation results demonstrate that the three methods, namely, the a) DFT, b) PSO and c) PSO-RLS methods, perform well and can estimate harmonic state percentages with the steady-state error, that is, $4.11 \%, 3.75 \%$ and $3.4 \%$, respectively. Figure 5 illustrates that the proposed computational PSO-RLS method is more accurate than the other two methods.

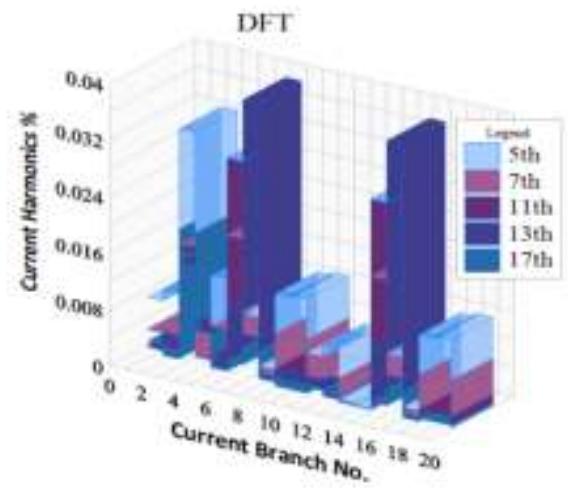

(a) DFT method

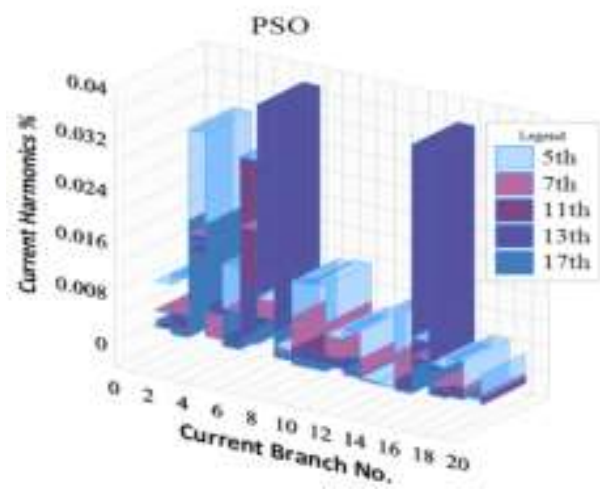

(b) PSO method

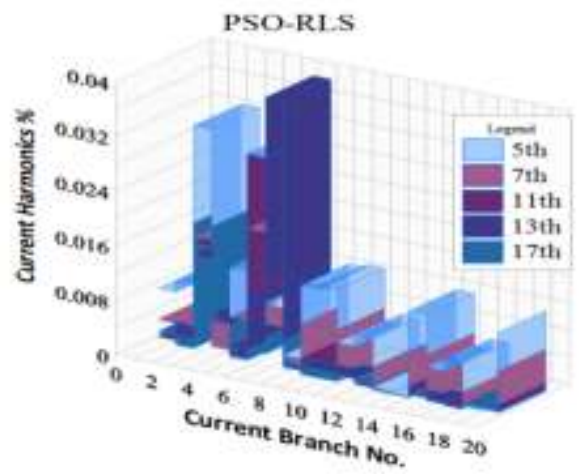

(c) PSO-RLS method

Figure 4. Current harmonics of each of the 14 IEEE buses; a) DFT method; b) PSO method; c) PSO-RLS method

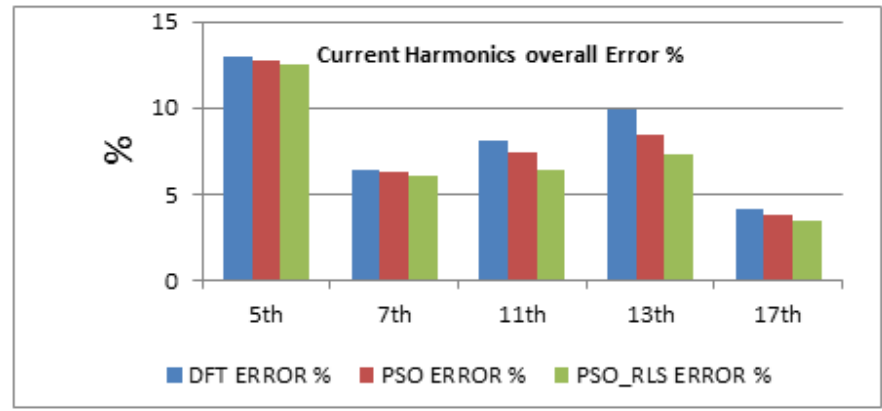

Figure 5. Overall current harmonic error 
Table 3. Voltage bus angle of 14 IEEE buses

\begin{tabular}{ccccc}
\hline Bus No. & Calculation & DFT & PSO & PSO_RLS \\
\cline { 1 - 5 } 1 & 0 & 0 & 0 & 0 \\
2 & -0.0785 & -0.0861 & -0.20188 & -0.0852 \\
3 & -0.21407 & -0.223 & -0.29067 & -0.22277 \\
4 & -0.1699 & -0.195 & -0.20745 & -0.1789 \\
5 & -0.22231 & -0.2321 & -0.23794 & -0.23091 \\
6 & -0.14413 & -0.1543 & -0.18416 & -0.1528 \\
7 & -0.24301 & -0.2461 & -0.27163 & -0.2515 \\
8 & -0.22231 & -0.2321 & -0.25706 & -0.23092 \\
9 & -0.24993 & -0.2653 & -0.2485 & -0.26853 \\
10 & -0.25363 & -0.26223 & -0.23705 & -0.25223 \\
11 & -0.25037 & -0.2797 & -0.20774 & -0.2598 \\
12 & -0.25785 & -0.2745 & -0.21501 & -0.2564 \\
13 & -0.2585 & -0.291 & -0.21958 & -0.2771 \\
14 & -0.27158 & -0.2848 & -0.23374 & -0.38018 \\
\hline
\end{tabular}

Table 4. Voltage bus angle error of 14 IEEE buses

\begin{tabular}{cccc}
\hline & DFT & PSO & PSO-RLS \\
\hline Overall Error \% & 0.21115 & 0.19014 & 0.17632 \\
\hline
\end{tabular}

\section{CONCLUSION}

In this study, a new algorithm for HSE in power systems is proposed, which is a hybrid between the PSO and RLS algorithms, namely, the PSO-RLS algorithm. The new method is compared with the two methods (i.e. the DFT and PSO methods), and the results show that the PSO-RLS algorithm has satisfactory accuracy and computational time as a state estimator for amplitudes and phases. The evaluation of the performance of the proposed algorithm (i.e. the PSO-RLS algorithm) compared with that of the DFT and PSO algorithms is reported. A comparison among the three algorithms (i.e. the DFT, PSO and PSO-RLS algorithms) shows that the proposed PSO-RLS algorithm is the best method. Moreover, based on the simulation results and analysis, the new proposed PSO-RLS work proves that it can be used as an estimator for harmonics in a power system.

\section{REFERENCES}

[1] Enayati, J., \& Moravej, Z., "Real-time harmonics estimation in power systems using a novel hybrid algorithm," IET Generation, Transmission \& Distribution, vol. 11, no. 14, pp. 3532-3538, 2017. doi:10.1049/iet-gtd.2017.0044.

[2] Z. Liu, Y. Xu, H. Jiang and S. Tao, "Study on Harmonic Impedance Estimation and Harmonic Contribution Evaluation Index," in IEEE Access, vol. 8, pp. 59114-59125, 2020, doi: 10.1109/ACCESS.2020.2982950.

[3] Zhang, J., Meng, Z., Gou, S., "Harmonic estimation using symmetrical interpolation FFT based on triangular selfconvolution window," IEEE Trans. Ind. Inf., 11, pp. 16-26, 2015, https://doi.org/10.1109/TII.2014.2362491.

[4] J. Sun, E. Aboutanios, D. B. Smith and J. E. Fletcher, "Robust Frequency, Phase, and Amplitude Estimation in Power Systems Considering Harmonics," in IEEE Transactions on Power Delivery, vol. 35, no. 3, pp. 1158-1168, June 2020, doi: 10.1109/TPWRD.2019.2936885.

[5] Beltran-Carbajal, F., Silva-Navarro, G., "A fast parametric estimation approach of signals with multiple frequency harmonics", Electr. Power Syst. Res., vol. 144, pp. 157-162, 2017, https://doi.org/10.1016/j.epsr.2016.11.023.

[6] W. Zhou, O. Ardakanian, H. Zhang and Y. Yuan, "Bayesian Learning-Based Harmonic State Estimation in Distribution Systems With Smart Meter and DPMU Data," in IEEE Transactions on Smart Grid, vol. 11, no. 1, pp. 832-845, Jan. 2020, doi: 10.1109/TSG.2019.2938733.

[7] Husham, I. H. and Ahmed, M. G., "A hybrid model for state estimation prediction composed of neural network and PSO algorithm for Iraqi national super grid system," First International Scientific Conference of Engineering Sciences-3rd Scientific Conference of Engineering Science (ISCES), pp. 50-55, 2018, doi: 10.1109/ ISCES.2018.8340527.

[8] Singh, S.K., et al., "Several variants of Kalman filter algorithm for power system harmonic estimation", Int. J. Electr. Power Energy Syst., vol. 83, pp. 793-800, 2016, https://doi.org/10.1016/j.ijepes.2015.12.028.

[9] Y. Xi et al., "Harmonic estimation in power systems using an optimised adaptive Kalman filter based on PSO-GA," IET Generation, Transmission \& Distribution, vol. 13, no. 17, pp. 3968-3979, 39 2019, doi: 10.1049/ietgtd.2018.6148.

[10] H. I. Hussein., Ghassan, A. S. and Mohammed, S.H., "Phase measurement units based FACT's devices for the improvement of power systems networks controllability," International Journal of Electrical and Computer Engineering (IJECE), vol. 8, pp. 888-899, 2018, doi: 10.11591/ijece. v8i2.

[11] Husham, I. H., "Neural network controller based Dstatcom for voltage sag mitigation and power quality issue," International Journal of Engineering and Technology, vol. 8, no. 1, pp. 405-420, 2016. 
[12] Raghad Ali Mejeed, Ahmed K. Jameil, and Husham Idan Hussein, "Harmonic Amplification Damping Using a DSTATCOM-Based Artificial Intelligence Controller," International Journal of Sensors, Wireless Communications and Control, vol. 9, no. 4, pp. 521-530, , 2019, DOI:10.2174/2210327909666190611142348.

[13] H. I. Hussein, Ghassan Abdullah Salman and Ahmed Majeed Ghadban, "Employment of PSO Algorithm to Improve the Neural Network Technique for Radial Distribution System State Estimation", International Journal on Smart Sensing And Intelligent Systems, vol. 12, no. 1, pp. 1-10, 2019, DOI: 10.21307/ijssis-2019-005.

[14] F. Ornelas-Tellez, J. J. Rico-Melgoza, R. Morfin-Magaña and S. Ramos-Paz, "Optimal Dynamic Harmonic Extraction and Suppression in Power Conditioning Applications," in IEEE Transactions on Industrial Electronics, vol. 67, no. 9, pp. 7909-7918, Sept. 2020, doi: 10.1109/TIE.2019.2944059.

[15] L. Chen, W. Zhao, F. Wang and S. Huang, "Harmonic Phasor Estimator for P-Class Phasor Measurement Units," in IEEE Transactions on Instrumentation and Measurement, vol. 69, no. 4, pp. 1556-1565, April 2020, doi: 10.1109/TIM.2019.2916961.

[16] S. Babaev, S. Cobben, V. Ćuk and H. van den Brom, "Online Estimation of Cable Harmonic Impedance in LowVoltage Distribution Systems," in IEEE Transactions on Instrumentation and Measurement, vol. 69, no. 6, pp. 2779-2789, June 2020, doi: 10.1109/TIM.2019.2926690.

[17] Z. Zhang, Z. Qu and J. Yang, "Optimal Harmonic Measuring Device Placement in Distribution Networks in Consideration of Topology Changes," in IEEE Access, vol. 8, pp. 85339-85347, 2020, doi: 10.1109/ACCESS.2020.2992678.

[18] C. F. M. Almeida and N. Kagan, "Harmonic state estimation through optimal monitoring systems," in IEEE Transactions on Smart Grid” vol 4, no. 1, pp. 467-478, 2013, doi: 10.1109/TSG.2012.2235472

[19] H. Ahmed, M. Bierhoff and M. Benbouzid, "Multiple Nonlinear Harmonic Oscillator-Based Frequency Estimation for Distorted Grid Voltage," in IEEE Transactions on Instrumentation and Measurement, vol. 69, no. 6, pp. 2817 2825, June 2020, doi: 10.1109/TIM.2019.2931065.

[20] Ghassan Abdullah Salman, Husham Idan Hussein, Mohammed Saadi Hasan, "Enhancement The Dynamic Stability of The Iraq's Power Station Using PID Controller Optimized by FA and PSO Based on Different Objective Functions", Elektrotehniški Vestnik, vol. 85, no. 1-2 pp. 42-48, 2018.

[21] Hayrettin Gokozan, Sezai Taskin, Serhat Seker, Huseyin EkizA, "Neural network based approach to estimate of power system harmonics for an induction furnace under the different load conditions", Electrical Engineering, vol. 97, no. 2, pp. 111-117, 2015, DOI 10.1007/s00202-014-0320-3.

[22] M. Comanescu, "Estimation of THD, Harmonic Components and Power Factor in Three-Phase Rectifiers," 2020 IEEE International Conference on Industrial Technology (ICIT), Buenos Aires, Argentina, pp. 468-473, 2020, doi: 10.1109/ICIT45562.2020.9067212.

[23] N. J. Li, et al., "Enhanced particle swarm optimizer incorporating a weighted particle," Neurocomputing, vol. 124, pp. 218-227, 2014, doi: 10.1016/j.neucom.2013.07.005.

[24] Ali N. A, Ahmed M. G., Hayder S. H., H. I. Hussein, "Enhancement the stability of power system using optimal location of FACTS devices", Indonesian Journal of Electrical Engineering and Computer Science (IJEECS), vol. 18, no. 2, pp. 648-655, 2020, DOI: 10.11591/ijeecs.v18.i2.pp648-655.

[25] Jamal Abdul-Kareem Mohammed, Arkan Ahmed Hussein, Sahar R. Al-Sakini. "Voltage disturbance mitigation in Iraq's low voltage distribution system," Indonesian Journal of Electrical Engineering and Computer Science (IJEECS), vol. 17, no. 1, pp. 47-60, January 2020, DOI: 10.11591/ijeecs.v17.i1.pp. 47-60.

[26] H. I. Hussein, A. I. Jaber, "Open Loop \&Closed Loop Vector Control Of VFD Three Phase Version Induction Motor Drives," Diyala Journal of Engineering Sciences, vol. 10, no. 2, pp. 27-38, 2017, https://doi.org/10.24237/djes.2017.10203.

[27] G. A. Salman, "Automatic Generation Control In Multi Area Interconnected Power System Using PID Controller Based On GA And PSO," Diyala Journal Of Engineering Sciences, vol.8, no.4, pp. 297 -310, 2015.

\section{BIOGRAPHIES OF AUTHORS}
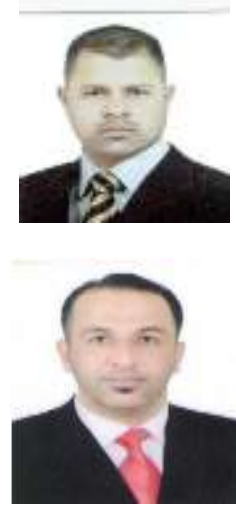

Hassan S. Naji was born in Baghdad, Iraq, in 1979, received his B.Sc. from University Diyala / Iraq in 2003, M.Sc. from University Baghdad in 2013. He is currently assistance lecturer in Department of Biomedical Engineering, College of Engineering, University of Kerbala, Iraq. Professional Strength and Skills: current his research interests are electronic and communication engineering, digital communication, filter design, artificial Inteligence AI.. He is teaching several basic subjects of the Biomedical Engineering, University of Kerbala- Iraq.

Husham I. Hussein was born in Baghdad, Iraq, in 1978, received his B.Sc. from University Technology/Iraq in 2002, M.Sc. from University Technology of Malaysia (UTM) in 2012. He is currently assistance professor in Department of Electrical Power and Machines Engineering, College of Engineering, University of Diyala, Iraq. Professional Strength and Skills: current his research interests are optimization power system, power quality, power system state estimation and Renewable Energy. He has good experience in practice of Electrical engineering in different fields such as installation of industrial plants and other electrical design and executing works. He is teaching several basic subjects of the Electrical Engineering, University of Diyala - Iraq. He isa member of IAENG. 